\title{
"Dewatering" of the lungs at birth
}

One of the most remarkable events to witness is the very rapid replacement of fluid by air in the normal neonatal lung at birth. Transformation to air breathing is so rapid that it is very difficult to envisage the normal physiological mechanisms for maintaining homeostasis in the air filled lung expelling so much fluid so rapidly-especially when, up to that point, those mechanisms have been shifting fluid in the reverse direction in utero.

Such rapid expulsion of aqueous fluid from porous media are commonly effected in the physical sciences by use of cationic surfactants termed "de-watering" agents. ${ }^{1}$ They function by chemisorption to the solid surface, reversibly bound by their polar moieties, to impart some degree of hydrophobicity and, hence, water repellency.

It has been suggested ${ }^{1}$ that, in the normal air filled lung, water repellency imparted by an adsorbed pseudo-cationic surfactant in the form of surface active phospholipid (SAPL), is responsible for alveolar fluid being confined to the "pools" observed ${ }^{2}$ in the septal corners, and "pits" elsewhere along what is otherwise an apparently fluid free surface. A continuous liquid layer would impair gas exchange.

This concept, however, conflicts with popular respiratory theory ${ }^{3}$ which, unlike the physical sciences, ${ }^{1}$ restricts the role of surfactant in the lung to the liquid-air interface where it reduces surface tension of the alveolus viewed as a one sided bubble. ${ }^{3}$ This "bubble" model gains its popularity from the fact that a liquid-air interface must dominate entry of the first breath into a lung initially filled with fluid. Moreover, exogenous surfactant formulated to spread rapidly over liquid-air interfaces is usually successful in rapidly establishing adequate gas exchange in infants afflicted with respiratory distress syndrome. ${ }^{4}$ However, events occurring some 48 hours later determine the ultimate outcome of "surfactant rescue," weaning from the ventilator being dependent on clearance of residual fluid. Thus "dewatering" offers a time frame consistent with the much slower rates for surfactants binding to solids than spreading over liquid surfaces. ${ }^{1}$

If this concept is essentially correct, then SAPL would need to be already in place, adsorbed to alveolar epithelium

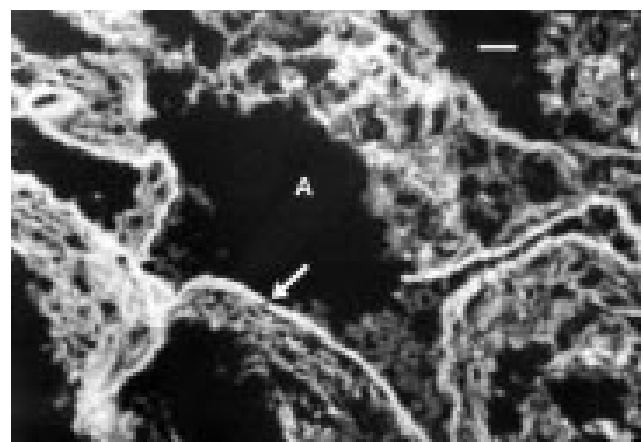

Figure 1 Photomicrograph of alveolar tissue excised from a stillborn human fetus at 41 weeks of gestation, taken by epifluorescence microscopy using Phospholipin $E$ as the hydrophobic probe. Note the lining layer bordering the potential air space $(A)$ : original magnification $\times 300$. before the onset of labour in a normal full term delivery for the transition to air-breathing to be so rapid.

If SAPL is already adsorbed to alveolar tissue in a normal mature fetus before delivery, then it should also be present in a stillborn human fetus of 41 weeks gestational age which had died of non-respiratory causes. At necropsy within 24 hours of the estimated time of death of this female stillborn, small $(2 \mathrm{~mm} \times 2 \mathrm{~mm} \times 2 \mathrm{~mm})$ samples of peripheral lung tissue were excised and immediately fixed for epifluorescence microscopy, using Phosphin E (Kasei, Tokyo; PO602), as the hydrophobic probe, as described in detail by Ueda et al..$^{5}$ Transverse sections of saline rinsed alveolar epithelium stained with Phosphin E were observed under the microscope (Nikon "Optiphot" with fluorescence attachment) under ultraviolet excitation in the range $450-490 \mathrm{~nm}$ with a barrier at $520 \mathrm{~nm}$. In all sections the alveolar epithelial surface displayed an intense green-gold colour characteristic of oligolamellar $\mathrm{SAPL}^{5}$ (fig 1).

Spectral analysis using a scanning spectrophotometer (Beckman DU64) of light emitted from the spot indicated in fig 1 gave a spectrum (fig 2) virtually identical with that of identically stained pure dipalmitoyl phosphatidylcholine (DPPC), shown by Bangham and Horne to produce oligolamellar SAPL when ultrasonicated in water. ${ }^{1}$ These results leave little doubt, if any, that the outermost layer lining fetal alveolar epithelium at term is SAPL, if not DPPC - a finding highly compatible with those of Ueda et $a \bar{l}$ for normal adult lung.

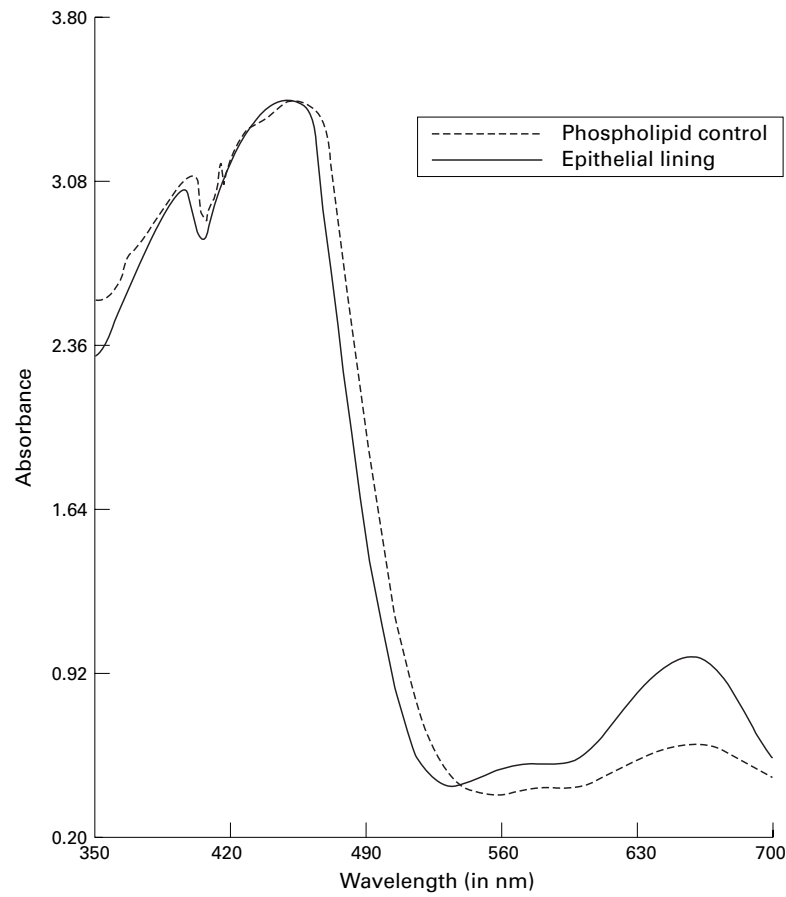

Figure 2 Spectral analysis of light (arrow in fig 1). Note identical spectrum from synthetic DPPC transformed into its oligolamellar state as liposomes. 
These findings have been repeated on three further stillborns (one girl of 41 weeks and two boys of 40 and 41 weeks of gestational age), although the SAPL lining was less pronounced in one boy in whom necropsy had been delayed for over 48 hours from the estimated time of death.

\section{Discussion}

The results must add strong support for the presence of SAPL already adsorbed to the alveolar epithelial surface of the normal neonate before birth. Thus it is ideally situated to effect "dewatering" just as commercial surfactants have been very widely used for similar purposes in vitro. ${ }^{1}$ Other examples of "dewatering" in vivo range from the cockroach cuticle to the human ocular surface where an adsorbed layer of SAPL is responsible for break up of the tear film. ${ }^{1}$

The fact that SAPL needs to be in its oligolamellar state to produce the spectrum shown in fig 2 is consistent with the lamellated alveolar lining so clearly shown in adult lungs by electron microscopy in the study by Ueda $e t a \bar{P}$ and confirmed by ourselves. Thus it is difficult to dismiss those elegant findings as artefact on the basis that tannic acid causes SAPL migration. ${ }^{6}$

The "adult" fluid profile of the alveolus is probably attained in normal neonates very rapidly. In the neonate with respiratory distress syndrome, however, it would take much longer to achieve even though exogenous surfactant designed to spread rapidly at a liquid-air interface may have established adequate oxygenation. Thus the vital "second stage" of surfactant rescue seems to involve much slower adsorption of SAPL to alveolar epithelium, explaining the need ${ }^{4}$ for diagnostic evidence of fluid retention to be acceptable before the neonate can be safely weaned from the ventilator. This has implications for the formulation of exogenous surfactant for "rescue," such that it not only spreads rapidly across the surface of incumbent water to admit air, but undergoes subsequent chemisorption to effect removal of that water.

1 Hills BA. The Biology of Surfactant. Cambridge: Cambridge University Press, 1988

2 Weibel ER, Limacher W, Bachofen H. Electronmicroscopy of rapidly frozen lungs: Evaluation on the basis of standard criteria. $f$ Appl Physiol 1982;53:516.

3 Hawgood S, Clements JA. Pulmonary surfactant and its proteins. $\mathcal{F}$ Clin Invest 1990;86:1-6.

4 Milner AD. How does exogenous surfactant work? Arch Dis Child 1993;68:253-4

5 Ueda S, Kawamura K, Ishii N, et al. Ultrastructural studies on surface lining layer of the lungs: Part IV. Resected human lungs. F fap Med Soc Biol Interface 1985;16:34-60.

6 Schrijvers AHGJ, Frederik PM, Stuart MCA, et al. Formation of multilamellar vesicles by addition of tannic acid to phosphatidylcholine containing small unilamellar vesicles. F Histochem Cytochem 1989;37:1635-43.

BRIAN A HILLS I BRENT MASTERS

Paediatric Respiratory Research Centre,

Department of Respiratory, Medicine,

Mater Children's Hospital,

South Brisbane, QD 4101,

Australia

\section{Commentary}

Two questions are raised by this paper: first, does pulmonary surfactant work in the way hypothesised so that it is able to "dewater" the lungs; and second, if it can, does it have a role in removing lung liquid at birth?

The paper is presented as further support of Professor Hill's "dry lung" theory of surfactant function-that is, pulmonary surfactant is applied directly on to the alveolar epithelium and any alveolar liquid exists as small droplets which lie on top of the surfactant lipid layer. Large hydrostatic pressures would be generated inside the droplets due to their small convex radii of curvature which would then drive the liquid through the epithelium and into the interstitium. This is an attractive idea, albeit devoid of strong supporting experimental evidence.

The photomicrograph presented shows that a layer of phospholipid is near the epithelial surface, but it cannot prove binding between the two. The resolution is insufficient to disprove that an aqueous layer lies between the epithelium and the phospholipid layer because calculated alveolar liquid layer thickness is of the order of $0.1 \mu \mathrm{m} .{ }^{1}$ Indeed, electron microscopy carefully performed by others has shown an alveolar layer of these dimensions. ${ }^{2}$

Furthermore, the surfactant complex has a net negative charge at alveolar $\mathrm{pH}(6-7)$. It is not cationic or even pseudocationic, and because the pulmonary epithelium has a mucopolysaccharide coat which is probably polyanionic, surfactant is unlikely to bind to the surface and to behave like industrial cationic detergents. ${ }^{3}$

Space does not permit adequate discussion of further evidence supporting the alternative traditional theory of surfactant function - such as the existence of an aqueous alveolar lining in which certain surfactant forms are suspended, and on which lies a surface active phospholipid layer so the reader is referred elsewhere. ${ }^{3}$

However, even if the "dry lung" theory were true, it would be unlikely to explain the lung liquid absorption which underlies transition to air breathing at birth. All fetal mammals secrete lung liquid at prodigious rates, but near term the pulmonary epithelium becomes increasingly sensitive to $\beta$ adrenergic stimulation which can cause the opening (or activation) of sodium channels on the luminal surface. This results in rapid removal of lung liquid from the lumen and into the interstitium. In vivo, this process is stimulated by adrenaline (and probably other agents, including vasopressin) which rises to sufficiently high levels even in early labour, so "dewatering" of the lung begins well before birth and before the establishment of an air-liquid interface in the alveoli. ${ }^{4}$ Evidence for the role of active ion transport in removing lung liquid at birth is overwhelming. Absence of sodium channels in the lung, a situation which has been genetically engineered in a "knock out" mouse model, results in an inability to remove lung liquid at birth and universal early death - and this in animals in whom there has been no interference with surfactant function. ${ }^{5}$

Thus the body of existing evidence does not support a "dewatering" action for pulmonary surfactant at birthintriguing and seductive though the suggestion is.

1 Stephens RH Benjamin AR, Walters DV. Volume and protein concentration in epithelial lining in perfused in situ postnatal sheep lungs. 7 Appl Physiol in epithelial lining

2 Bastacky J, Lee CY, Goerke J, et al. Alveolar lining layer is thin and continuous: low temperatiure scanning electron microscopy of rat lung. $\mathcal{F} A p p l$ Physiol 1995;79:1615-28.

3 King RJ, Clements JA. Surface active material from dog lung. II. Composition and physiological correlations. Am F Physiol 1972;223:715-26.

4 Strang LB. Fetal lung liquid secretion and reasbsorption. Physiol Rev 1991;71:991-1016.

5 Hummler F, Barker P, Gatzy J, et al. Early death due to defective neonatal lung liquid clearaance in alpha $\mathrm{ENaC}$ deficient mice. Nature Genet 1996;12:325-8.

Department of Child Health

D V WALTERS

St George's Hospital Medical School

Cranmer Terrace

London SW17 ORE 